\title{
Surface Inhomogeneities on the W UMa star AC Bootis
}

\author{
Albert P. Linnell \\ Department of Physics and Astronomy, Michigan State University, \\ E. Lansing, MI 48824, USA
}

\begin{abstract}
New photometric data and two older data sets for AC Boo can be represented with a common set of Roche model parameters and two starspot pairs which drift only in longitude and are located on the cooler component.
\end{abstract}

The W-type W UMa star AC Bootis is well known for the variability of its light curve. A persistent light curve feature is an O'Connell effect with the maximum following primary minimum (max.I) brighter than the one preceding primary minimum (max.II). Existing light synthesis studies have not modeled this feature, and they have been hampered by lack of a cross-correlation radial velocity determination of the mass ratio.

In a collaborative effort, B. Hrivnak has obtained a cross-correlation spectroscopic mass ratio $q^{*}\left(q^{*}=1 / q\right)$ of 0.35 (preliminary value). E. C. Olson has observed a precise $435.5 \mathrm{~nm}$ light curve (1989 data). Although the O'Connell effect can be represented by a single dark spot on the advancing face of the more massive component, a second persistent feature cannot be so represented. This feature is a displacement of max.I to orbital phase 0.27 . An eccentric orbit is an unacceptable possible explanation. In the absence of a persuasive argument for bright starspots, the max.I displacement must be the result of depression of the light curve egress from primary minimum by dark spot(s). To do this, starspots must be on the trailing face of the more massive component. The O'Connell effect then becomes a relatively delicate competition between spots separated in longitude by roughly 180 degrees, but differing somewhat from that value.

Experiment demonstrates that the synthesized light curve is relatively insensitive to spot latitude in the range 0-25 degrees. In an effort to minimize the number of free parameters, and by analogy to the sun, the light curve simulation adopted two spot pairs at latitudes $(+20,-20$ degrees) and with fixed temperature contrast from the adjacent photosphere of $-1500 \mathrm{~K}$. Both spots of a given pair have identical longitudes.An accurate fit to the Olson data, using the Hrivnak mass ratio, required adoption of 8 percent third light. 
Having achieved a precise fit to the Olson data, it is of interest whether other data sets can be represented with the same system of parameters, varying only the spot longitudes. The data sets tested included the $V$ data by Manusco et al. (1976) and the $U B V$ data by Schieven et al. (1983). An accurate fit resulted in each case. The additional data sets required a small admixture of third light approximating that required by the Olson data. The difference in longitude of the spot pairs was maintained constant while changing the spot longitudes to fit the two additional data sets. It is of interest that the O'Connell effect was absent in the Manusco et al. data, while the Schieven et al. data show an O'Connell effect opposite in sense to the Olson data. Moreover, the Schieven et al. data show a positive light curve slope during total eclipse which the light synthesis simulation represents accurately.

The Olson data were obtained over an interval of about two months, in two separate observing sessions. Plots of the separate sessions show a slight systematic change on ingress to primary minimum, but with coincident egress. The later session ingress is slightly brighter, at all phases, than the earlier one. Based on the adopted longitude of the spot pair that produces the O'Connell effect, the light curve change indicates that the spot pair drifted forward on the star, implying a faster than synchronous rotation at the starspot latitudes. Since the light curve change persists into totality, when the less massive component is eclipsed, the observations unequivocally place the spots on the larger, more massive component. A detailed joint paper with B. Hrivnak and E. C. Olson is in preparation describing AC Boo.

\section{References}

Manusco, S., Milano, L., Russo, G.: 1978, Astron. Astrophys. 63, 193

Schieven, G., Morton, J. C., McLean, B.J., Hughes, V.A.: 1983, Astron. Astrophys. Suppl. Ser. 52, 463 